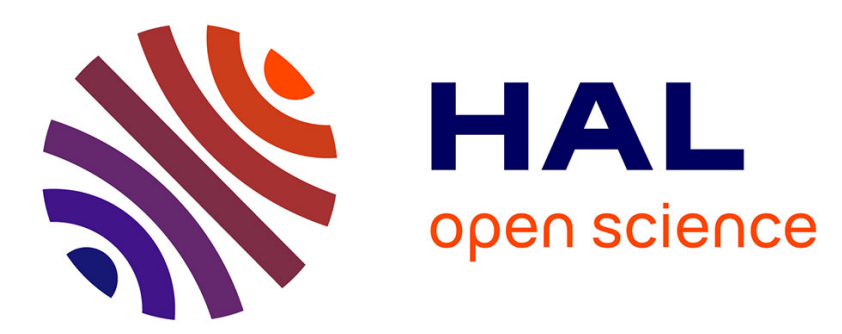

\title{
Energy Modeling of Wireless Body Area Networks with On-Body Communication Channel Characterization
}

\author{
Amina Nahali, Abdelaziz Hamdi, Matthieu Gautier, Antoine Courtay, Rafik
}

Braham

\section{- To cite this version:}

Amina Nahali, Abdelaziz Hamdi, Matthieu Gautier, Antoine Courtay, Rafik Braham. Energy Modeling of Wireless Body Area Networks with On-Body Communication Channel Characterization. International Wireless Communications \& Mobile Computing Conference (IWCMC), Jun 2019, Tanger, Morocco. hal-02184476

\section{HAL Id: hal-02184476 https://hal.inria.fr/hal-02184476}

Submitted on 16 Jul 2019

HAL is a multi-disciplinary open access archive for the deposit and dissemination of scientific research documents, whether they are published or not. The documents may come from teaching and research institutions in France or abroad, or from public or private research centers.
L'archive ouverte pluridisciplinaire HAL, est destinée au dépôt et à la diffusion de documents scientifiques de niveau recherche, publiés ou non, émanant des établissements d'enseignement et de recherche français ou étrangers, des laboratoires publics ou privés. 


\title{
Energy Modeling of Wireless Body Area Networks with On-Body Communication Channel Characterization
}

\author{
Amina Nahali*, Abdelaziz Hamdi*, Matthieu Gautier ${ }^{\dagger}$, Antoine Courtay ${ }^{\dagger}$, Rafik Braham* \\ ${ }^{*}$ PRINCE Research Laboratory, ISITC, University of Sousse, Tunisia, ${ }^{\dagger}$ Univ Rennes, CNRS, IRISA, France \\ amina.nahali@irisa.fr, abdelaziz.hamdi@polymtl.ca, matthieu.gautier@irisa.fr, antoine.courtay@irisa.fr, dr.rafik.b@ieee.org
}

\begin{abstract}
Wireless sensor systems represent reliable platforms for monitoring and managing of a variety of applications for health care, civil, and military environments. As most of these systems are energy constrained, an accurate control of the energy consumption is required to well design the system. The main focus of this paper is the accurate energy modeling of Wireless Body Area Networks. To this end, we discuss the propagation channel model at $2.4 \mathrm{GHz}$ between two body sensors placed on the human body. This model combines path losses in free space and on human body. Then, an analytical energy consumption model is derived by including channel characteristics such as the proposed path loss model, small scale fading and signal to noise ratio. The model is validated by measurements performed with patch antennas on human subjects. Our results provide interesting insights about the effect of on-body propagation on the global path losses. Moreover, with the proposed approach, the correlation between propagation channel features and energy efficiency is highlighted.
\end{abstract}

Index Terms-Wireless Body Area Networks, path loss, energy model, channel modeling, measurements, electromagnetic simulations.

\section{INTRODUCTION}

Wireless Body Area Networks (WBANs) can be viewed as an extension to Wireless Sensor Networks (WSNs). A WBAN consists of a set of miniature sensors integrated in/on or near the human body. The WBAN architecture can be subdivided into three communication levels as presented in Fig. 1. These communication levels are defined as tier-1: intraWBAN communication, tier-2: inter-WBAN communication, and tier-3: beyond-WBAN communication [1]. Intra-WBAN communication depicts the interaction between body sensor nodes, tier-2 presents the communication between the sensor nodes and the coordinator node, and the beyond-WBAN communication explicitly presents the service offered by the WBAN technology. For example, for a medical application use case, a database containing information about individual health, identification of diseases and medical operations is mandatory.

Intra-BAN communications use the human body as propagation medium. Therefore, some attenuation and mitigation can affect the signal quality, so that the human body behaves as a lossy medium. The characterization of the physical layer of the network is an important step in the development of a WBAN, including its energy consumption and the path loss between two nodes on the body. Propagation channel modeling and antenna design have been investigated in [2][12] in different applicative scenarios: in-body/on-body and off body at different frequencies.

In [2] and [5], the authors have developed a BAN model based on human phantom properties. Thus, they solve the propagation equations around a cylinder and an ellipsoid geometry characterizing an approximation of the body form. These models are validated by using full-wave electromagnetic simulations and measurement compaigns. Other research results [6], [11] have focused on path loss, shadowing and multipath characterization inside the human body for different scenarios. On the other hand, some works [3], [12] apply traditional path loss measurements to perform the channel gain between transmitter and received devices. All the previous research studies mentioned above have only focused on developing or designing a model that is invarious to communication conditions. Even though these research works take into account some features related to human body morphology, frequency and communication mode, they are not interested in propagation channel model utility in WBAN systems. To this end, several other works [13], [14] propose cross layer approaches. The aim is to use physical propagation analysis to investigate protocol level application in order to optimize network topology in terms of energy efficiency.

In this paper, we propose a new propagation model based on link budget evaluation and path attenuation between two nodes placed on the human body by taking into consideration both path losses due to the radio communication and human body medium at $2.4 \mathrm{GHz}$. Measurements campaigns were performed in an indoor environment on a human body subject to validate the theoretical channel formulation. In addition, a new energy consumption model is derived by incorporating the proposed model.

This paper is organized as follows: Section II presents the state of the art related to the characterization of the physical layer including both communication channel and energy efficiency design for WBANs. In Section III, the proposed onbody propagation model is presented by including human body path loss. In Section IV, the energy consumption model based on these propagation conditions is derived. Section V first presents experimental investigations to validate the theoretical models and discusses measurement results concerning the received power fluctuations and simulation results concerning 


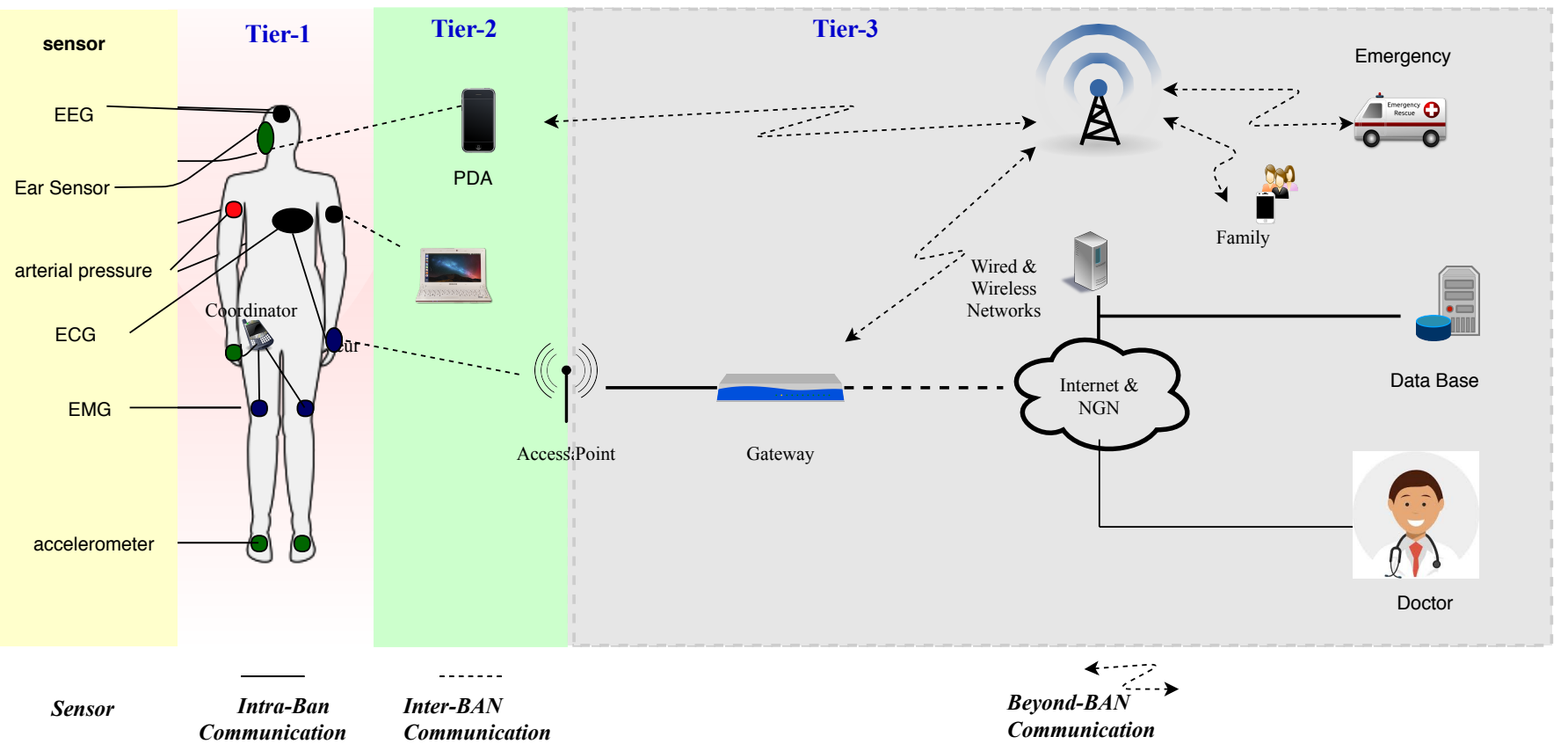

Fig. 1. Communication Tiers in a WBAN for a medical application use case.

the energy consumption. Section VI concludes the paper.

\section{RELATED WORKS}

In the past decade, WBANs have become an extremely active field of research in both WSN and engineering communities in the aim to investigate the energy efficiency in relation with propagation signal and wireless communication channel. In [15], a radio energy model for WBAN was proposed. This model uses the distance $\mathrm{d}$ between the transmitter namely Nordic nF 2401A and the receiver namely Chipcon CC2420 used for WBAN and assumes the loss of energy due the transmission channel. The authors propose a relay network in which, each sensor has a relay node in its line of sight (LOS) and connects to this transition network by one short hop. This relay network uses an energy-efficient protocol to access the channel and route the information. In [16], the energyaware WBAN design model was improved to minimize the energy consumption providing a new energy efficient topology. In this approach, relay nodes are placed off the body to help the sensor nodes forwarding data to the sink. Although this approach is interesting, it fails to take into account the propagation channel conditions. In [17], an analytical expression of energy efficiency was derived by introducing channel specification including signal to noise ratio (SNR) and distance based path loss. However, in this study, there is still a need for considering the body posture influence in the path loss estimation. In [18], the authors propose an approach to optimize the system level energy efficiency by selecting different strategies. This approach considers the threshold distance which is responsible for the proportion of transmission energy and circuit energy consumption in
WBAN. Nevertheless, the channel characterization is limited to large scale effects including the path loss phenomenon due merely to traditional wireless communication; i.e. path losses due the human body posture are ignored. In [13], the authors propose an analytical expression of the energy consumption by including small scale fading and large scale fading. They present a relevant energy consumption model based on the communication link SNR in order to propose a new cross layer based on joint network and physical layer and improve the routing algorithm by considering the energy saving issued by the analytical expression. Otherwise, this model was derived for wireless sensor networks application not for body sensor networks. Unlike wireless sensor networks environment when the network size is an important property, a WBAN is a small network. In [19], an energy efficient model during communication between sensors is proposed. The authors presented a multi-hop topology model which uses a relay between the coordinator and distant sensor nodes. Based on this proposed topology, the WBAN energy efficient model was developed. Simulation results showed that lower energy is used when a relay node is considered in the network topology.

As mentioned above, several research papers have studied the impact of the channel conditions by exploiting path losses and multipath effects on the energy efficiency. Nevertheless, they are based on the energy consumption model that considers only path loss fading depending on both distance and frequency and ignoring the human body effects. In other words, the human body is complex and its tissues have electrical attributes which affect the communication of electromagnetic signals emitted from these nodes [20]. In order to optimize 
energy consumption, WBAN needs to employ low power transmission, these signals are however absorbed by human tissues. As a result, the process of data retransmission occurs and eventually affects the energy consumption. In the next section, we first propose a new path loss model introducing the whole path attenuation including free space and body absorption losses. Then, an energy consumption model based on this new analytical formulation is proposed.

\section{MODEL FOR ON-BODY PROPAGATION}

In order to predict wave propagation in WBAN environment, the following scenario is considered: two antennas as transceiver/receiver are placed on the human body at $2.4 \mathrm{GHz}$ [21]. In fact, electromagnetic waves propagate as creeping waves when the considered human body parts present a curved path or as surface waves when the considered parts are flat surfaces. In this paper, all physical parameters in the channel modeling are considered, including the effect of the propagation medium; free space and human body. An analytical formulation to predict on-body channel model at $2.4 \mathrm{GHz}$ is proposed. A possible model can be extracted starting from the most general free space equation. As compared to the free space link budget, the appearance of the human tissue medium introduces an additional effect on the propagation wave. This effect can be presented by the body morphology attenuation. Introducing the received and transmitted powers $P_{R x}$ and $P_{T x}$ and the two antenna gains $G_{R x}$ and $G_{T x}$, an on-body channel model can be expressed by:

$$
P_{R x_{(d B m)}}=P_{T x_{(d B m)}}+G_{T x_{(d B i)}}+G_{R x_{(d B i)}}-A_{p_{(d B)}} .
$$

where $A_{p}$ is the relevant factor that can be inevitable and necessary to optimize the link budget between $T x-R x$ antennas. Then $A_{p}$ must containing the dependence in term of frequency, distance, and propagation medium attenuation and can be expressed by the sum of a free-space component $A_{F S}$ and a human body component $\alpha_{B}$.

\section{A. Path-loss model in free-space}

According to friis formula, the path loss in the free space propagation medium can be easily determined as:

$$
L_{0_{(d B)}}=20 \log (4 \pi / c)
$$

with $c=3.10^{8} \mathrm{~m} / \mathrm{s}$ the celerity of light.

$L_{0}$ is equal to $92.44 \mathrm{~dB}$ at $2.4 \mathrm{GHz}$. The expression of the path-loss in the free-space can be written as:

$$
A_{F S_{(d B)}}=92.44+20 \log (f)+20 \log (d) .
$$

\section{B. Path-loss model in human-body}

In electromagnetic characterization, the human body is considered as a complex medium. This medium has different permittivity $\varepsilon_{r}$ and conductivity $\sigma$. Thus, propagating wave produces some electromagnetic effects with body tissues and body posture such as energy absorption, reflection, diffraction and shadowing. In this work, these effects can be modeled as

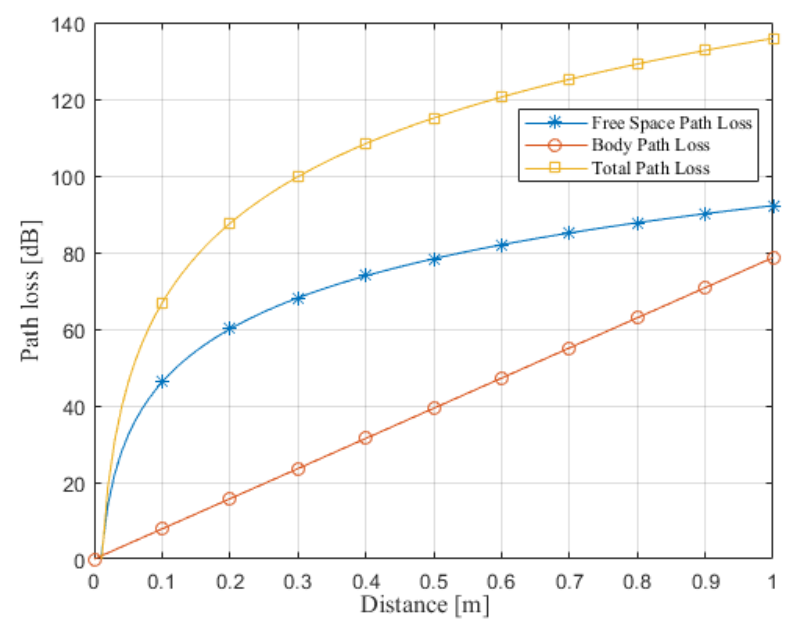

Fig. 2. Path loss VS distance.

attenuation $\alpha_{B}$ that can be written in $\mathrm{Np} / \mathrm{cm}$ in (4) [4], with $\sqrt{\frac{\mu_{0}}{\epsilon_{0}}}$ is the free space impedance.

$$
\alpha_{B_{(N p / m)}} \approx \frac{\sigma}{2} \sqrt{\frac{\mu_{0}}{\epsilon_{0} \epsilon_{r}}} .
$$

Considering that $T x$ and $R x$ are separated by $25 \mathrm{~cm}$, the attenuation expression $\alpha_{B}$ converted in $\mathrm{dB}$ is:

$$
\alpha_{B_{(d B)}} \approx \frac{520.8 \pi \sigma}{\sqrt{\epsilon_{r}}} .
$$

At $2.4 \mathrm{GHz}$ frequency, electric parameters of human body are: 50 for permittivity $\left(\varepsilon_{r}=50\right)$ and $1.7 \mathrm{~S} / \mathrm{m}$ for conductivity $(\sigma=1.7 \mathrm{~S} / \mathrm{m})$ [22].

\section{Total path-loss of the proposed model}

The total path-loss $A_{p}$ in (1) is then equal to the sum of the amounts of the $A_{F S}$ and $\alpha_{B} . A_{p}$ can be written as:

$$
A_{p_{(d B)}}=92.44+20 \log (f)+20 \log (d)+\frac{520.8 \pi \sigma}{\sqrt{\epsilon_{r}}} .
$$

The multipath caused by the environment around the body is also considered as one of the factors producing fading which makes the WBAN channel modeling different from the ones in other environments. Fig. 2 shows the developed path loss model (6) as a function of the distance between $T x / R x$ antennas on the human body when the antennas are aligned. Path loss increases of course when the antennas separation increases. In comparison with the free space medium, path loss on the human body is more important. For example, at $2.4 \mathrm{GHz}$, the free space path loss is equal to $80 \mathrm{~dB}$ when the antennas are separated by $50 \mathrm{~cm}$. whereas, the total path loss is equal to $120 \mathrm{~dB}$. A $40 \mathrm{~dB}$ loss is therefore due to the human body medium. 


\section{ON-BOdy CHANNEL PROPAGATION BASED ENERGY CONSUMPTION MODEL}

WBAN consumes energy during sensing, communication and data processing. As much energy is consumed during communications, the proposed model specifies the latter one specifically during signal propagation. In order to evaluate the energy consumption system in WBAN environment, the propagation channel characteristics are considered. Many researches are focusing on the traditional path loss due to $T x-R x$ distance which is very simple and does not reflect the reality of the propagation environment [15]. In our proposed energy model, we assume two types of consumed energy: the transmission energy per bit denoted by $E_{T x}$ depending on the distance $d$ and the reception energy per bit $E_{R x}$. The model equations are:

$$
\begin{gathered}
E_{T x}=E_{\text {elec }}+E_{a m p} d^{\alpha}, \\
E_{R x}=E_{\text {elec }},
\end{gathered}
$$

$E_{\text {elec }}$ is the energy required by the circuitry of transmitter and receiver in $\mathrm{J} / \mathrm{bit}$ and $E_{a m p}$ is the energy required by the amplifier circuit in $\mathrm{J} / \mathrm{bit}, d$ is the distance between the transmitter node and the receiver node. $\alpha$ is the path loss factor defined by the propagation environment. Reliability of the body channel is a multi-faceted criterion and not only the function of channel fading. In our context, small scale fading (multipath fading), body morphology attenuation and large scale fading (path loss) are taken into account to ensure a reliable communication. According to our proposed model, the latter attenuation is modeled by $\alpha_{B}$. Seeing that our proposed model was empirically validated, the propagation phenomenon factor can be included in the energy consumption model to predict a relevant energy model based on realistic conditions. From physical layer point of view, the received power at a destination over the distance $d$ due to the path loss is expressed by:

$$
\frac{P_{R x}}{P_{T x}}=\frac{G_{T x} G_{R x} \lambda^{2}}{(4 \pi)^{2}(d)^{\alpha}} .
$$

By considering the path attenuation due to the human body loss $\alpha_{B}$, the received power can be expressed as

$$
P_{R x}=\frac{P_{T x} G_{T x} G_{R x} \lambda^{2}}{(4 \pi)^{2} d^{\alpha}} e^{-\alpha_{B} d}=\frac{K}{d^{\alpha}} e^{-\alpha_{B} d} .
$$

$\alpha_{B}$ is done by (5), $\alpha$ is the path loss factor; $\alpha \in[3,4]$ for LOS WBAN and $\alpha \in[5,7.4]$ for NLOS WBAN [6]; finally $K=\frac{G_{t} G_{r} \lambda^{2}}{(4 \pi)^{2}}$. Since in WBAN, there is attenuation due to human body absorption, a body loss coefficient parameter $\alpha_{B}$ is added to (8), it can be written as:

$$
E_{T x}=E_{e l e c}+E_{a m p} \alpha_{B} d^{\alpha} .
$$

By considering the small scale fading characterized by the multipath $|h|$, the received power $P_{R x}$ can be expressed as:

$$
P_{R x}=P_{T x} \frac{K}{d^{\alpha}}|h|^{2} .
$$

Thus, the SNR can be expressed as:

$$
S N R=\frac{P_{T x} K}{N_{0} B d^{\alpha}}|h|^{2},
$$

where $N_{0}$ is the noise spectral density, and $B$ is the reception filter bandwidth. Thus, for a given SNR over the same channel conditions, the required transmit energy per bit $E_{T x}$ (11) is expressed as:

$$
E_{T x}=E_{\text {elec }}+\frac{S N R \cdot N_{0} B}{R_{b} k|h|^{2}} d^{\alpha} \alpha_{B},
$$

where $R_{b}$ is the bit rate in bit/s. From (14), it is worth noticing that the energy consumption depends not only on the source destination distance but also on the source destination channel attenuation including the SNR, the multipath fading and the body path attenuation. Based on the proposed WBAN system illustrated in Fig. 3, we make the assumption that the radio propagation channel is symmetric such that the energy required to transmit a message from node $T x$ to node $R x$ is the same as the energy required to transmit a message from node $R x$ to node $T x$.

\section{RESUlts AND Discussion}

\section{A. Experimental validation}

In order to validate the proposed model, experiments were performed using a radio communication plateform. For the signal acquisition, the Agilent 89600 vector signal analyzer was used and synchronized with an N5182A signal generator as a transmitter. The transmitting power $P_{T x}$ is set to 0 $\mathrm{dBm}$ (the same used in [4] [23] [24] and $15 \mathrm{dBm}$ was used in [25]). The frequency center was set to $2.4 \mathrm{GHz}$, with 1001 points. One pair of patch antennas $(T x$ and $R x)$ is placed at various positions on the human body having vertical polarization (Fig. 3). During the measurement campaign, the antennas were placed parallel to each other and lined up for maximal power transfer. In order to adjust the antennas on the body, a transparent film roll was fixed and flexible cables with an SMA connector were used to facilitate the body movements. The measurements were performed on a female person; 25 years old. The transmitting antenna $T x$ is placed at a fixed position on the wrist and the receiving antenna $R x$ is moved along various positions on the arm towards $80 \mathrm{~cm}$. The wireless on-body channel for LOS indoor environment along a stretched arm was examined. Fig. 4 shows a comparison between the theoretical model and measurements results. The distance between the antennas varies from $0 \mathrm{~cm}$ to $80 \mathrm{~cm}$ in step of $5 \mathrm{~cm}$. For each measurement point, a total of 50 values were recorded for the time-varying electric field and the average received power was calculated for a fixed $2.4 \mathrm{GHz}$ frequency. Our experimental setup bears a good match to the analytical formulation when $d$ is less than $40 \mathrm{~cm}$, from 40 to $80 \mathrm{~cm}$ it becames less accurate. In fact, from $35 \mathrm{~cm}$ an abrupt change of channel is noticed with minimum amplitude of 6 $\mathrm{dB}$ and maximum amplitude of $13 \mathrm{~dB}$. 


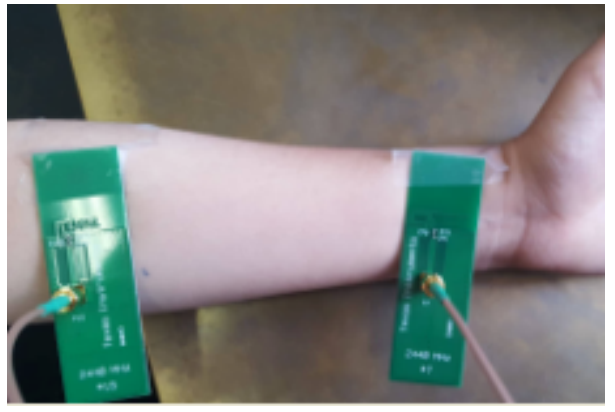

Fig. 3. On-Body measurement setup.

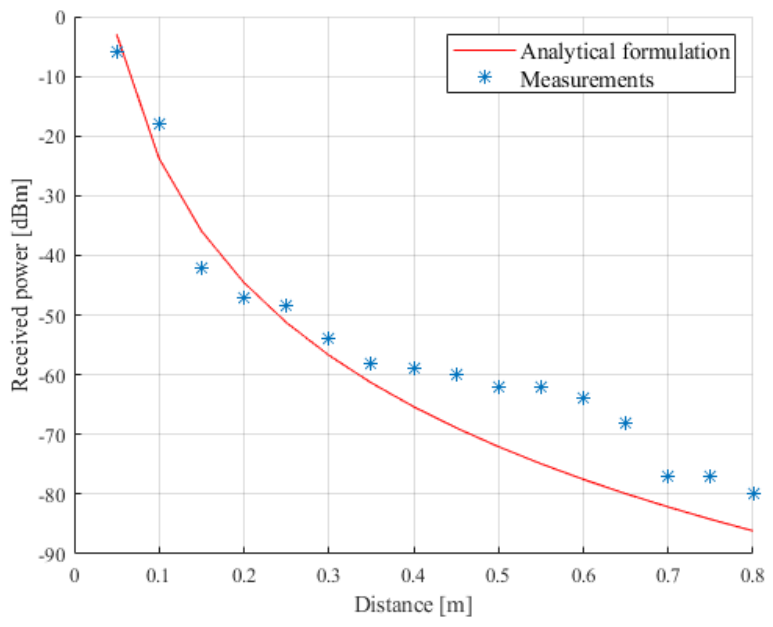

Fig. 4. Analytical and measured received power on the human body at $2.4 \mathrm{GHz}$.

\section{B. Energy consumption}

The energy consumption variations are investigated by Finite Integration Technique simulation in CST-MS. Fig. 5 shows the variation of energy consumption expended in the system as the network diameter increases from $0.2 \mathrm{~m}$ to $2 \mathrm{~m}$. For our simulations, we also assume that transmitter $T x$ and receiver $R x$ are sensing the environment at a fixed rate and thus always have data to send to the end-user. To illustrate this point, we consider the linear network, where the distance between the nodes is $d$. The energy expended in the radio electronics $E_{\text {elec }}$ increases until $25 \mu \mathrm{J} / \mathrm{bit}$, for the scenario where each node has a 2000 bit data packet to send to the $R x$ station. This shows that, as montionned by our analysis previously, when transmission energy is on the same order as receive energy, which occurs when transmission distance is short and/or the radio electronics energy is high, direct transmission is more energy efficient than the minimum of the transmitted energy. Moreover, Fig. 5 clearly reveals the variation of the transmitted energy $E_{T x}$ and received energy $E_{R x}$ at $2.4 \mathrm{GHz}$. The transmitted energy $E_{T x}$ is fixed to be $500 \mu \mathrm{J}$. The energy received $E_{R x}$ is significantly decreased from the $250 \mu \mathrm{J}$ to $10 \mu \mathrm{J}$, with distance $d$ variation from 0

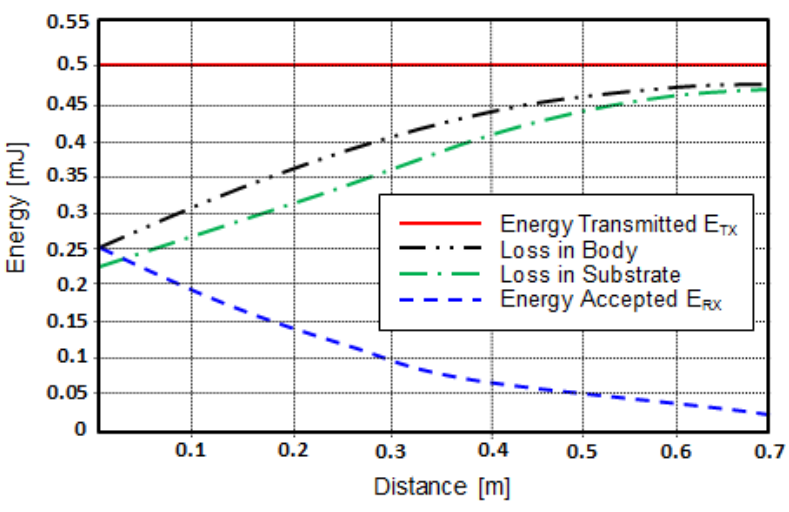

Fig. 5. Energy consumption variations at $2.4 \mathrm{GHz}$.

to $0.7 \mathrm{~m}$. This verifies our assumptions and will allow more accurate evaluation of different WBAN scenarios.

\section{CONCLUSION}

In this paper, a path loss formulation taking into consideration path attenuation as well as attenuation due to human body posture was presented. We have demonstrated that the human body has a rigorous influence on the propagation model as well as the energy efficiency. Path loss increases certainly when the antennas separation increases and in the presence of the human body and it is more important than free space medium. A propagation model was proposed analytically and validated experimentally and an appropriate energy model was given for WBAN conditions. Based on simulation results, we conclude that, a relevant energy consumption model is obtained by adding all propagation channel characteristics. Our proposed path loss model is able to predict on-body propagation at $2.4 \mathrm{GHz}$ and subsequently able to model energy efficiency under these conditions.

\section{REFERENCES}

[1] S. Movassaghi, M. Abolhasan, J. Lipman, D. Smith, and A. Jamalipour, "Wireless body area networks: A survey," IEEE Communications Surveys \& Tutorials, vol. 16, no. 3, pp. 1658-1686, 2014.

[2] T. Mavridis, L. Petrillo, J. Sarrazin, D. Lautru, A. Benlarbi-Delai, and P. De Doncker, "Theoretical and experimental investigation of a 60ghz off-body propagation model," IEEE transactions on antennas and propagation, vol. 62, no. 1, pp. 393-402, 2014.

[3] T. Chrysikos, I. Zisi, and S. Kotsopoulos, "Channel modeling and path loss characterization for in-body propagation at MICS and ISM bands,' in IEEE Wireless Telecommunications Symposium (WTS), 2016, pp. 1-7.

[4] T. Alves, B. Poussot, and J.-M. Laheurte, "Analytical propagation modeling of BAN channels based on the creeping-wave theory," IEEE Transactions on Antennas and Propagation, vol. 59, no. 4, pp. 12691274, 2011.

[5] R. Chandra and A. J. Johansson, "An elliptical analytic link loss model for wireless propagation around the human torso," in IEEE European Conference on Antennas and Propagation (EUCAP), 2012, pp. 31213124.

[6] K. Y. Yazdandoost, K. Sayrafian-Pour et al., "Channel model for body area network (BAN)," IEEE 802.15.6 technical contribution, document ID: 15-08-0780-09-0006, pp. 41-56, 27 April 2009.

[7] A. Taparugssanagorn, A. Rabbachin, M. Hämäläinen, J. Saloranta, J. Iinatti et al., "A review of channel modelling for wireless body area network in wireless medical communications," 2008. 
[8] H. Viittala, M. Hämäläinen, and J. Iinatti, "Different experimental WBAN channel models and IEEE802. 15.6 models: comparison and effects," in IEEE International Symposium on Applied Sciences in Biomedical and Communication Technologies, (ISABEL), 2009, pp. 1-5.

[9] T. Kumpuniemi, M. Hamalainen, J.-P. Makela, and J. Iinatti, "Path loss modeling for uwb creeping waves around human body," in IEEE International Symposium on Medical Information and Communication Technology (ISMICT), 2017, pp. 44-48.

[10] R. Braham, F. Douma, and A. Nahali, "Medical body area networks: Mobility and channel modeling," in IEEE International Conference on Science of Electronics, Technologies of Informations and Telecommunications (SETIT), 2016, pp. 1-6.

[11] K. SaiSanathKumar, K. N. K. Reddy, V. Pushpavathy, P. R. Reddy, D. Sharma, P. K. Sharma, and B. V. Krishna, "Calculation of Path Losses at CM3 for Wireless Body Area Networks (WBAN) by using Different Types of Antennas," International Journal of Applied Engineering Research, vol. 11, no. 7, pp. 5210-5217, 2016.

[12] D. B. Smith and L. W. Hanlen, "Channel modeling for wireless body area networks," in Ultra-Low-Power Short-Range Radios. Springer, 2015, pp. 25-55.

[13] A. B. Ammar, A. Dziri, M. Terre, and H. Youssef, "Cross-layer approach based energy minimization for wireless sensor networks," Wireless Personal Communications, vol. 98, no. 2, pp. 2211-2221, 2018.

[14] G.-M. Hoang, M. Gautier, and A. Courtay, "Cooperative-cumconstrained maximum likelihood algorithm for uwb-based localization in wireless bans," in IEEE International Conference on Communications (ICC), 2015, pp. 2704-2709.

[15] E. Reusens, W. Joseph, B. Latré, B. Braem, G. Vermeeren, E. Tanghe, L. Martens, I. Moerman, and C. Blondia, "Characterization of on-body communication channel and energy efficient topology design for wireless body area networks," IEEE Transactions on Information Technology in Biomedicine, vol. 13, no. 6, pp. 933-945, 2009.

[16] A. Rostampour, N. Moghim, and M. Kaedi, "A new energy-efficient topology for wireless body area networks," Journal of medical signals and sensors, vol. 7, no. 3, p. 163, 2017.

[17] M. Waheed, R. Ahmad, W. Ahmed, M. Drieberg, and M. M. Alam, "Towards efficient wireless body area network using two-way relay cooperation," Sensors, vol. 18, no. 2, p. 565, 2018.

[18] C. Yi, L. Wang, and Y. Li, "Energy efficient transmission approach for WBAN based on threshold distance," IEEE sensors journal, vol. 15, no. 9, pp. 5133-5141, 2015.

[19] R. C. Chepkwony, "Energy Efficient Model for Deploying Wireless Body Area Networks Using Multi-Hop Network Topology." Ph.D. dissertation, 2017.

[20] G. Ragesh and K. Baskaran, "An overview of applications, standards and challenges in futuristic wireless body area networks," International Journal of Computer Science Issues (IJCSI), vol. 9, no. 1, p. 180, 2012.

[21] A. Nahali, A. Hamdi, and R. Braham, "Body area networks: Path loss modeling and antenna design," in IEEE International Wireless Communications and Mobile Computing Conference (IWCMC), 2018, pp. $174-179$.

[22] P. S. Hall, Y. Hao, Y. I. Nechayev, A. Alomainy, C. C. Constantinou, C. Parini, M. R. Kamarudin, T. Z. Salim, D. T. Hee, R. Dubrovka et al., "Antennas and propagation for on-body communication systems," IEEE Antennas and Propagation Magazine, vol. 49, no. 3, pp. 41-58, 2007.

[23] S. J. Ambroziak, L. M. Correia, R. J. Katulski, M. Mackowiak, C. Oliveira, J. Sadowski, and K. Turbic, "An off-body channel model for body area networks in indoor environments," IEEE Transactions on Antennas and Propagation, vol. 64, no. 9, pp. 4022-4035, 2016.

[24] M. M. Khan, Q. H. Abbasi, M. Rahman, and R. H. Ashique, "Experimental study of on-body radio channel performance of a compact ultra wideband antenna," Journal of Electromagnetic Analysis and Applications, vol. 7, no. 1, pp. 1-9, 2015.

[25] J. A. Ruiz, J. Xu, and S. Shimamoto, "Propagation characteristics of intra-body communications for body area networks," in Consumer Communications and Networking Conference, 2006. CCNC 2006. 3rd IEEE, vol. 1. IEEE, 2006, pp. 509-513. 